\title{
Boson Mott insulators at finite temperatures
}

\author{
Fabrice Gerbier \\ Laboratoire Kastler Brossel, ENS, Université Pierre et Marie-Curie-Paris 6, \\ CNRS ; 24 rue Lhomond, 75005 Paris, Franc屯
}

(Dated: October 23, 2018)

\begin{abstract}
We discuss the finite temperature properties of ultracold bosons in optical lattices in the presence of an additional, smoothly varying potential, as in current experiments. Three regimes emerge in the phase diagram: a low-temperature Mott regime similar to the zero-temperature quantum phase, an intermediate regime where MI features persist, but where superfluidity is absent, and a thermal regime where features of the Mott insulator state have disappeared. We obtain the thermodynamic functions of the Mott phase in the latter cases. The results are used to estimate the temperatures achieved by adiabatic loading in current experiments. We point out the crucial role of the trapping potential in determining the final temperature, and suggest a scheme for further cooling by adiabatic decompression.
\end{abstract}

PACS numbers: 03.75.Lm,03.75.Hh,03.75.Gg

Ultracold gases in optical lattices are currently a major topic in the field of ultracold atoms, both experimentally and theoretically (see the reviews [1, 2, 3, 4]. So far, experiments are mostly carried out with Bose gases, and interpreted in terms of the zero-temperature phase diagram of the Bose-Hubbard model [1, 3, 4]. However, actual experiments inevitably take place at finite temperatures, which leads, e.g., to residual number fluctuations. In view of its importance for applications such as the controlled generation of entangled states [1], or the study of quantum magnetism 3], this issue has recently received increasing interest [5, 6, 7, 8, 9, 10, 11, 12, 13]. In this paper, we first discuss how the phase diagram of a ultracold Bose gas in an optical lattice is modified at finite temperatures. We identify a melting temperature $T^{*} \approx 0.2 U / k_{\mathrm{B}}$, above which the system is entirely thermal, and a much lower critical temperature $T_{\mathrm{c}} \approx z J$, above Mott regions survive, but superfluidity is absent. Here $z$ is the coordination number, $U$ the on-site interaction energy, and $J$ the tunneling energy. We derive analytically the thermodynamics of the Mott phase for $T<T^{*}$, including particle-hole corrections. Finally, we model the procedure adopted in current experiments to produce quantum gases in optical lattices, where a BoseEinstein condensate is slowly loaded into the optical potential in the presence of an "external", smoothly varying trap. Assuming the loading is done adiabatically, we estimate the final temperatures that can be reached with this procedure. We point out the crucial role of the external potential in determining the final temperature, and suggest a scheme for further cooling by adiabatic decompression.

For future reference, we first recall the phase diagram for zero temperature. For $J=0$, the ground state is a product of identical Fock states, with $n_{0}$ atoms at each site depending on the chemical chemical potential $\mu$ according to $n_{0}=\operatorname{Integer}(\mu / U)+1$. When a weak tunneling between nearest neighbors is allowed, the ground

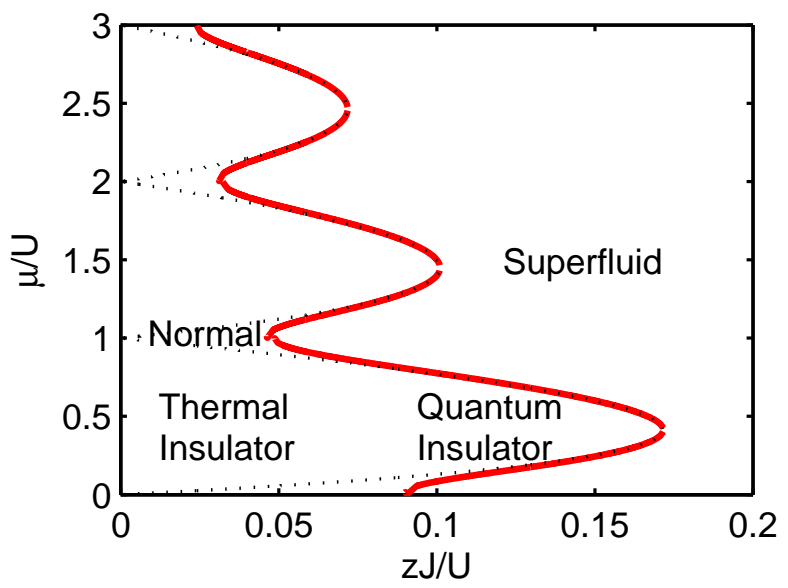

FIG. 1: Phase diagram for an ultracold Bose gas in an optical lattice at finite temperatures $\left(k_{B} T=U / 20\right)$. The finitetemperature Mott lobes boundaries are shown as solid lines, and the zero-temperature boundaries as dotted lines for comparison. With increasing temperature, the superfluid regions in between the Mott lobes shrink in width and shift to larger tunneling energies. For temperatures $z J<T$, the SF-MI transition is replaced by a smooth crossover between two Mott domains across a normal phase. This generic phase diagram persists up to the melting temperature $T^{*} \approx 0.2 U$ where the Mott domains vanish.

state remains a Mott insulator with suppressed number fluctuations but only in well-defined domains ("Mott lobes") in the $J-\mu$ plane (dashed lines in Fig. 1). Outside these domains, the MI is unstable towards a delocalized, superfluid state (SF), that connects to a condensate in the Bloch state $\mathbf{q}=\mathbf{0}$ when $J \gg U$. In current experiments, an external harmonic potential of the form $V_{\mathrm{T}}(\mathbf{r})=\frac{1}{2} m \omega_{\mathrm{T}}^{2} r^{2}$ is usually superimposed on the lattice potential. As a result, the density profile is in general not uniform everywhere, but has the shape of a "wedding cake" formed by successive layers ("Mott shells") with integer densities [14]. If this potential varies slowly 
over a few lattice sites, the local density approximation (LDA) can be employed. It obtains the (coarse-grained) number $n_{\mathrm{h}}[\mu, T]$ and entropy $s_{\mathrm{h}}[\mu, T]$ densities calculated as in the homogeneous case, but for a local chemical potential $\mu_{0}-V_{\mathrm{T}}(\mathbf{r})=\mu_{\text {loc }}(\mathbf{r})$. In the following, we first compute the phase diagram for uniform systems, and use the LDA to describe trapped gases.

Within a Mott domain with $n_{0}$ bosons per site, the lowest-lying excited states are "particle" and "hole" states, where a supplementary particle is added (respectively removed) to the background value $n_{0}$ with a free energy cost $U n_{0}-\mu\left(\right.$ resp. $\left.\sim \mu-U\left(n_{0}-1\right)\right)$. Other occupation numbers correspond to free energies at least of order $U$, and are therefore suppressed by a thermal factor $\sim$ $e^{-\beta U} \ll 1$. This allows to use what we call the "particlehole approximation" (PHA) 8, 9, 15, 16, 17, 18], which consists in truncating the on-site Hilbert space to the states $n_{0}, n_{0}-1$ and $n_{0}+1$. As we will see, this approximation, valid for temperatures $k_{B} T \ll U$, allows to describe quite extensively the physics of the insulating phase at finite temperatures.

As a starting point, we first neglect tunneling between the wells completely and consider the thermodynamics of a uniform system of isolated wells, where the atoms interact locally through the on-site interaction. Since the wells are independent, the global partition function factorizes into a product of identical on-site partition functions $z_{0}=\sum_{n} \exp [-\beta(E(n)-\mu n)]$, where $E(n)=U n(n-1) / 2$ and $\beta=1 / k_{\mathrm{B}} T$. Keeping only the particle/hole excitations with energies $E_{0}^{(\mathrm{qp})}=U n_{0}$ and $E_{0}^{(\mathrm{qh})}=U\left(n_{0}-1\right)$, the mean density and variance of density fluctuations take the simple forms

$$
\begin{aligned}
\bar{n}_{0} & \approx n_{0}+\left(B^{(+)}-B^{(-)}\right) / z_{0}, \\
\operatorname{Var}(\mathrm{n})_{0} & \approx\left(B^{(+)}+B^{(-)}\right) / z_{0}^{2},
\end{aligned}
$$

where $B^{(+)}=e^{\beta\left[\mu-U n_{0}\right]}$ and $B^{(-)}=e^{\beta\left[U\left(n_{0}-1\right)-\mu\right]}$ are Boltzmann factors corresponding respectively to adding or removing a particle from the "background" value $n_{0}$, and where $z_{0}=1+B^{(+)}+B^{(-)}$. In Fig. 2, we have shown the density and fluctuations as a function of the chemical potential. With increasing temperatures, the step-like density profile characteristic of a Mott state becomes increasingly smoother, reflecting the fact that the free energy cost to create supplementary particles or holes vanishes near the edges of a Mott plateau. A Mott-like region survives up to $T^{*} \sim 0.2 U / k_{B}$ (see also [7]), which can be seen as a melting temperature for the Mott phase. We have also compared the exact expressions to the PHA, without noticeable difference up to $T^{*}$.

With the properties of the zero-tunneling model clarified, we reintroduce tunneling in a second step. At zero temperature, when exploring a Mott domain at constant $J$ and varying chemical potential, the Mott state remains stable as long as its elementary (quasi-particle and hole) excitations are. At the upper or lower Mott lobe bound-
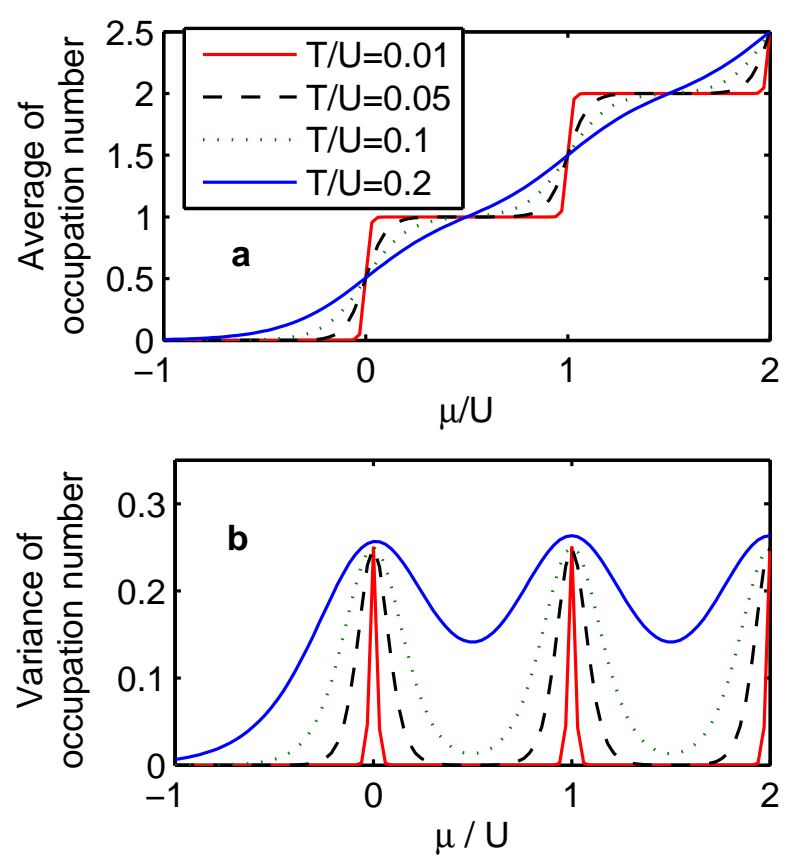

FIG. 2: Density profile and fluctuations for an array of isolated wells $(J=0)$, shown for various temperatures.

aries, the chemical potential $\mu^{( \pm)}$becomes equal to the excitations energies $E_{\mathbf{k}=0}^{(\mathrm{qp} / \mathrm{qh})}$, thus favoring the proliferation of particle or hole excitations to reduce the free energy [19]. To investigate how this behavior changes with increasing temperature, we have calculated the quasiparticles and holes dispersion relations at finite temperature using the PHA supplemented by a random-phase approximation as in [5, 20, 21, 22]. We find

$$
E_{\mathbf{k}}^{(\mathrm{qp} / \mathrm{qh})}=\frac{C J_{\mathbf{k}}}{2}+U\left(n_{0}-\frac{1}{2}\right) \pm \Delta_{\mathbf{k}}
$$

with $\Delta_{\mathbf{k}}=\sqrt{C^{2} J_{\mathbf{k}}^{2}+2 U D J_{\mathbf{k}}+U^{2}}, J_{\mathbf{k}}=-2 z J_{\chi_{\mathbf{k}}}$ and $\chi_{\mathbf{k}}=\frac{1}{z} \sum_{i=1} \cos \left(k_{i} d\right)$. The finite temperature dispersion relations are formally similar to the zero temperature expressions 20] except for the factors $C / D=$ $\left(n_{0}+1\right) A^{(\mathrm{qp})} \mp n_{0} A^{(\mathrm{qh})}$, with $A^{(\mathrm{qp} / \mathrm{qh})}=\left(1-B^{( \pm)}\right) / z_{0}$.

In the following, we mostly work for simplicity in the limit $U \gg z J$, where the dispersion relations reduce to $E_{\mathbf{k}}^{(\mathrm{qp} / \mathrm{qh})} \approx E_{0}^{(\mathrm{qp} / \mathrm{qh})} \mp J^{(\mathrm{qp} / \mathrm{qh})} \chi_{\mathbf{k}}$, with an effective tunneling energy $J^{(\mathrm{qp})}=z J\left(n_{0}+1\right) A^{(\mathrm{qp})}$ for particles and $J^{(\mathrm{qh})}=z J n_{0} A^{(\mathrm{qh})}$ for holes. We concentrate first on the upper boundary and try to find the chemical potential for which $E_{\mathbf{k}=0}^{(\mathrm{qp})}=\mu^{(+)}$. Introducing a variable $s=\left(U n_{0}-\mu^{(+)}\right) / k_{B} T$, we find that, up to terms $\sim e^{-\beta U}, s$ solves the equation $s-a \tanh \left(\frac{s}{2}\right)=0$, with $a=z J\left(n_{0}+1\right) / k_{B} T$. Graphic inspection shows that there is no solution for $a<2$, corresponding to a critical temperature $k_{B} T_{c}=z J\left(n_{0}+1\right) / 2$ above which there is no SF region left due to thermal depletion. This argu- 
ment holds far from the zero-temperature Mott transition, where Eqs. (31) are valid. To obtain the complete phase boundaries, we have solved numerically the equations $E_{\mathbf{k}=0}^{(\mathrm{qp} / \mathrm{qh})}=\mu^{( \pm)}$for any $z J / U$. The result is shown in Fig. 1, where three distinct regions emerge. For $z J \ll 2 k_{B} T /\left(n_{0}+1\right)$, one goes continuously from one insulating "lobe" to the other through a region in the normal phase. For $2 k_{B} T /\left(n_{0}+1\right) \ll z J \ll z J_{c}$, welldefined Mott lobes survive, as in the ground state, and above the critical $J_{c}$ a phase transition takes place to a SF phase, albeit with reduced SF fraction. Above $T \sim T^{*}$, we expect the system to be essentially normal for $J<U$. Note that when Mott regions with different filling factors due to an external trap potential, the regimes described above can coexist.

To characterize the thermodynamics for $T<T^{*}$ more quantitatively, we calculate the free energy as $F=F_{0}+\Delta F^{(\mathrm{qp})}+\Delta F^{(\mathrm{qh})}$. The first term $F_{0}=$ $-k_{B} T \ln \left(1+B^{(+)}+B^{(-)}\right)$gives the free energy corresponding to the thermal activation of local defects in each well for zero tunneling. The remaining contributions $\Delta F^{(\mathrm{qp})}=F_{\mathrm{BE}}\left[E_{\mathrm{k}}^{(\mathrm{qp})}-\mu\right]-F_{\mathrm{BE}}\left[U n_{0}-\mu\right]$ and $\Delta F^{(\mathrm{qh})}=F_{\mathrm{BE}}\left[\mu-E_{\mathbf{k}}^{(\mathrm{qh})}\right]-F_{\mathrm{BE}}\left[\mu-U\left(n_{0}-1\right)\right] \mathrm{de}-$ scribe instead dilute gases of quasi-particles and quasiholes mobile through the lattice. Here, the functional $F_{\mathrm{BE}}[x]=\sum_{\mathbf{k}} \ln (1-\exp (\beta x))$ gives the free energy of an ideal Bose gas. For $U \gg J$, we obtain for instance

$$
\left.\frac{\Delta F^{(\mathrm{qp})}}{N_{s} k_{B} T}=-\sum_{m=0}^{\infty} \frac{e^{m \beta\left(\mu-U n_{0}\right)}}{m}\left[I_{0}\left(\frac{m \beta J^{(\mathrm{qp})}}{\mathcal{D}}\right)^{\mathcal{D}}-1\right] 4,\right)
$$

and a similar expression for $\Delta F^{(\mathrm{qh})}$. Here $I_{0}$ is a Bessel function, and $\mathcal{D}$ the dimensionality.

Thermodynamic quantities follow from derivatives of the free energy. Contributions from local activation, denoted by the index " " have been calculated above. Terms coming from particle and hole contributions are readily obtained. For instance, the particle/hole corrections to the average density read $\Delta \bar{n}^{(\mathrm{qp} / \mathrm{qh})}=$ $-\partial \Delta F^{(\mathrm{qp} / \mathrm{qh})} / \partial \mu$, whereas the density fluctuations can be characterized using the Hellmann-Feynman theorem [23], which to leading order in $e^{-\beta U}$, reduces to $\operatorname{Var}(n) \approx$ $\operatorname{Var}(n)_{0}+\Delta \bar{n}^{(\mathrm{qp})}\left(1-\Delta \bar{n}^{(\mathrm{qp})}\right)-\Delta \bar{n}^{(\mathrm{qh})}\left(1+\Delta \bar{n}^{(\mathrm{qh})}\right)$.

In the low-temperature limit, $T \ll T_{c}$, the contribution of local activation terms is negligible, and tunneling corrections to the thermodynamic functions dominate. However, using the asymptotic form for $I_{0}$ we find for instance $\Delta F^{(\mathrm{qp})} / N_{s} k_{B} T=\left[k_{B} T / 4 \pi J\left(n_{0}+\right.\right.$ $1)]^{3 / 2} g_{5 / 2}\left(e^{\beta\left(\mu-\mu^{(+)}\right)}\right)$, where $g_{\alpha}(x)=\sum_{n} x^{n} / n^{\alpha}$ denotes a Bose function. The prefactor implies that corrections to the zero-temperature behavior are highly suppressed. Hence this regime correspond to a quantum Mott insulator, where the properties of the zerotemperature system are essentially preserved up to small thermal corrections. Increasing the temperature, corrections from local activation and from tunneling terms become progressively comparable until the high temperature regime, $T \gg T_{c}$, is reached. In this regime, using the Taylor expansion $I_{0}(x) \approx 1+x^{2} / 4$ for the Bessel function, we find

$$
\frac{\Delta F^{(\mathrm{qp})}}{N_{s} k_{B} T} \approx-\frac{1}{4 \mathcal{D}}\left(\frac{J\left(n_{0}+1\right)}{k_{B} T}\right)^{2} \frac{B^{(+)}}{z_{0}^{2}} .
$$

Note that this expression also applies to the normal phase in-between two Mott-like regions. Here the right hand side has a small term $\propto\left(J n_{0} / k_{B} T\right)^{2} \ll 1$, times a factor comparable to the $J=0$ result. This means that in this temperature regime, the model with $J=0$ alone already gives a good description of the thermodynamics.

As explained above, the results can be directly applied to the harmonically trapped case in the LDA. We apply now the calculation to estimate the temperatures achieved in current experiments. Typically, a BoseEinstein condensate is first produced in a magnetic trap with frequency $\omega_{i}$, at an initial temperature $T_{i}$. We calculate its entropy using the Popov approach as exposed in 24] using the local density approximation and for typical experimental values $\omega_{i}=2 \pi \times 20 \mathrm{~Hz}$ and $N=2 \times 10^{5}$ atoms. Then, the cloud is slowly transferred in the optical lattice of depth $V_{0}$. This also changes the frequency $\omega_{f}$ of the external harmonic potential according to

$$
\omega_{f}=\sqrt{\omega_{i}^{2}+\frac{8 V_{0}}{m w^{2}}} .
$$

In Eq. (6) the second term comes from the trapping force due to the Gaussian shape of the laser beams forming the lattice, with $w$ the lattice laser waist ( $1 / e^{2}$ radius), typically $\approx 150 \mu \mathrm{m}$. Assuming that the transfer into the lattice is adiabatic, both entropy and atom number are conserved. In the final state, assumed to be deep in the MI regime, the number density $n=-\partial f / \partial \mu$ and entropy density $s=-\partial f / \partial T$ are calculated from the free energy density $f$ [25]. Those densities are then integrated over space to fix the total atom number and entropy, which determines the final chemical potential and final temperature $T_{f}$. We plot in Fig. 3 a the result of a self-consistent calculation solving for $T_{f}$ for a fixed atom number $N=2 \times 10^{5}$. One sees that for small initial temperature $T_{i}<0.4 T_{\mathrm{c} 0}$ (condensed fraction larger than 75 $\%$ ) one ends well within the Mott region. Only by starting from a rather large thermal fractions does the final temperature exceed $0.2 \mathrm{U}$. Reaching the quantum insulator regime is more challenging, but still within reach of current experimental possibilities $\left(T_{i}<0.2 T_{\mathrm{c} 0}\right)$.

For this calculation, the role of the trapping potential is essential. Similar calculations for homogeneous systems with integer filling would yield a much higher temperature 10], since near the center of the Mott lobe the entropy is exponentially suppressed by the finite interaction energy $U$. In the trapped case, the entropy in 
fact concentrates around the lobe boundaries, where the generation of excitations is easiest. As long as $T<T^{*}$, the peak value of the entropy density is $s_{\max } \approx k_{\mathrm{B}} \log (2)$. The thickness $\delta R$ of the layer where thermal fluctuations are important can be estimated from $m \omega_{\mathrm{T}}^{2} R_{0} \delta R \sim k_{\mathrm{B}} T$, where $R_{0}=\sqrt{2 U / m \omega_{\mathrm{T}}^{2} d^{2}}$ is a typical spatial scale for the shell structure. Since the total entropy and atom number scale respectively as $S / k_{\mathrm{B}} \sim 4 \pi R_{0}^{2} \delta R$ and $N \sim(4 \pi / 3)\left(R_{0} / d\right)^{3 / 2}$, we find that in the trappe case, the entropy per particle rises linearly with temperature, $S / N k_{\mathrm{B}} \sim 3 k_{\mathrm{B}} T / U$, instead of exponentially $\left(\sim e^{-U / k_{\mathrm{B}} T}\right)$ as in the uniform case (see also [12] for a similar analysis).

An important consequence of Eq. (6) is that the trap frequency increases significantly when the lattice depth is raised. This leads to spatial compression (and the formation of Mott plateaux with higher filling), but also to adiabatic heating of the cloud, as in a conventional harmonic trap. In the second calculation, this effect was investigated [26]. In Fig. 3b, the result is plotted for a fixed initial temperature $T_{i}=0.3 T_{\mathrm{c} 0}$, but varying final trap frequencies. It is clear that lower and lower temperatures are achieved when the external trap frequency is reduced. In particular, one can achieve $T<0.01 U$ if the transfer is done at constant trap frequency. Experimentally, this could be realized using blue-detuned lattice beams, or additional blue-detuned (repulsive) lasers to compensate for the trapping force of the laser beams. Note finally the bump in the curve, that indicates that the transition from a unity-filled MI to a two-plateaux distribution with either single or double occupancy.

In conclusion, we have discussed the thermodynamics of ultracold bosons in optical lattices. We have identified two characteristic temperatures, $T \sim T_{c}$, above which the SF regions disappear, and a melting temperature $T^{*} \approx$ $0.2 U$. The thermodynamic function for the Mott phases are calculated explicitly. We have used the results to estimate the temperature reached in current experiments, and found that they easily reach the thermal insulator regime $T<T^{*}$, and possibly the quantum region $T<$ $T_{c}$. We suggest an adiabatic decompression scheme which potentially allows to reach much lower temperatures in a system with unity filling.

I would like to thank Immanuel Bloch, Jean Dalibard, Simon Fölling, Dries van Oosten and Artur Widera for discussions and comments. I acknowledge support from IFRAF and ANR.

* Electronic address: fabrice.gerbier@lkb.ens.fr

[1] D. Jaksch and P. Zoller, Annals of physics 315, 52 (2005).

[2] O. Morsch and M. Oberthaler, Rev. Mod. Phys. 78, 179 (2006).

[3] M. Lewenstein, A. Sanpera, V. Ahufinger, B. Damski, A. S. De, and U. Sen, cond-mat/0606771 (2006).
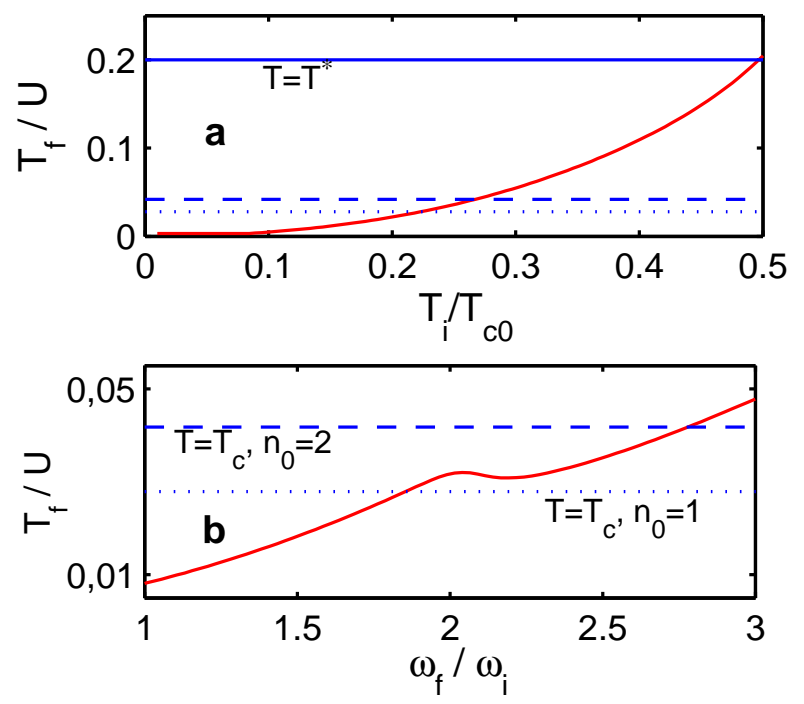

FIG. 3: Final temperature achieved by adiabatically transferring a condensate into the optical lattice potential (depth $V_{0}=20 E_{\mathrm{R}}$ ). The transfer is done in (a): for a fixed final trap frequency $\omega_{f}=2 \pi \times 70 \mathrm{~Hz}$ varying the initial temperature $T_{i}$, and in (b): for a fixed initial temperature $T_{i}=0.3 T_{\mathrm{c} 0}$ but varying the final trap frequency $\omega_{f}$. The solid line indicates the melting temperature $T^{*}$. Above the dashed and dotted lines, the superfluid layers corresponding to $n_{0}=2$ and $n_{0}=1$, respectively, turn normal. For both plots, the initial frequency $\omega_{i}=2 \pi \times 20 \mathrm{~Hz}$ and the atom number $N=2 \times 10^{5}$ are fixed.

[4] M.-B. P. with Ultracold Gases, cond-mat/0704.3011 (2007).

[5] D. B. M. Dickerscheid, D. van Oosten, P. J. H. Denteneer, and H. T. C. Stoof, Phys. Rev. A 68, 043623 (2003).

[6] L. Plimak, M. K. Olsen, and M. Fleischhauer, Phys. Rev. A 70, 013611 (2004).

[7] B. DeMarco, C. Lannert, S. Vishveshwara, and T.-C. Wei, Phys. Rev. A 71, 063601 (2005).

[8] A. M. Rey, G. Pupillo, and J. V. Porto, Phys. Rev. A 73, 023608 (2006).

[9] G. Pupillo, C. J. Williams, and N. V. Prokof'ev, Phys. Rev. A 73, 013408 (2006).

[10] K. P. Schmidt, A. Reischl, and G. S. Uhrig, Eur. Phys. J. D 38, 343 (2006).

[11] B. Capogrosso-Sansone, E. Kozik, N. Prokof'ev, and B. Svistunov, Phys. Rev. A 75, 013619 (2007).

[12] T.-L. Ho and Q. Zhou, Phys. Rev. Lett. 99, 120404 (2007).

[13] X. Lu and Y. Yu, Phys. Rev. A 74, 063615 (2006).

[14] S. Fölling, A. Widera, T. Müller, F. Gerbier, and I. Bloch, Phys. Rev. Lett. 97, 060403 (2006).

[15] D. Rokshar and G. Kotliar, Phys. Rev. B 44, 10328 (1991).

[16] W. Krauth, M.Caffarel, and J.-P. Bouchaud, Phys. Rev. B 45, 3137 (1992).

[17] E. Altman and A. Auerbach, Phys. Rev. Lett. 89, 250404 (2001).

[18] F. Gerbier, A. Widera, S. Fölling, O. Mandel, T. Gericke, and I. Bloch, Phys. Rev. A 72, 053606 (2005). 
[19] N. Elstner and H. Monien, Phys. Rev. B 59, 12184 (1999).

[20] D. van Oosten, P. van der Straten, and H. T. C. Stoof, Phys. Rev. A 63, 053601 (2001).

[21] D. M. Gangardt, P. Pedri, L. Santos, and G. V. Shlyapnikov, Phys. Rev. Lett. 96, 040403 (2006).

[22] K. Sengupta and N. Dupuis, Phys. Rev. A 71, 033629 (2005).

[23] K. V. Kheruntsyan, D. M. Gangardt, P. D. Drummond, and G. V. Shlyapnikov, Phys. Rev. Lett. 91, 040403 (2003).

[24] S. Giorgini, L. P. Pitaevskii, and S. Stringari, Phys. Rev. A 54, R4633 (1996).

[25] For this calculation, tunneling corrections are calculated using Eq. (5) rather than the full Eq. (41).

[26] This effect was independently noticed and analyzed in 12]. 

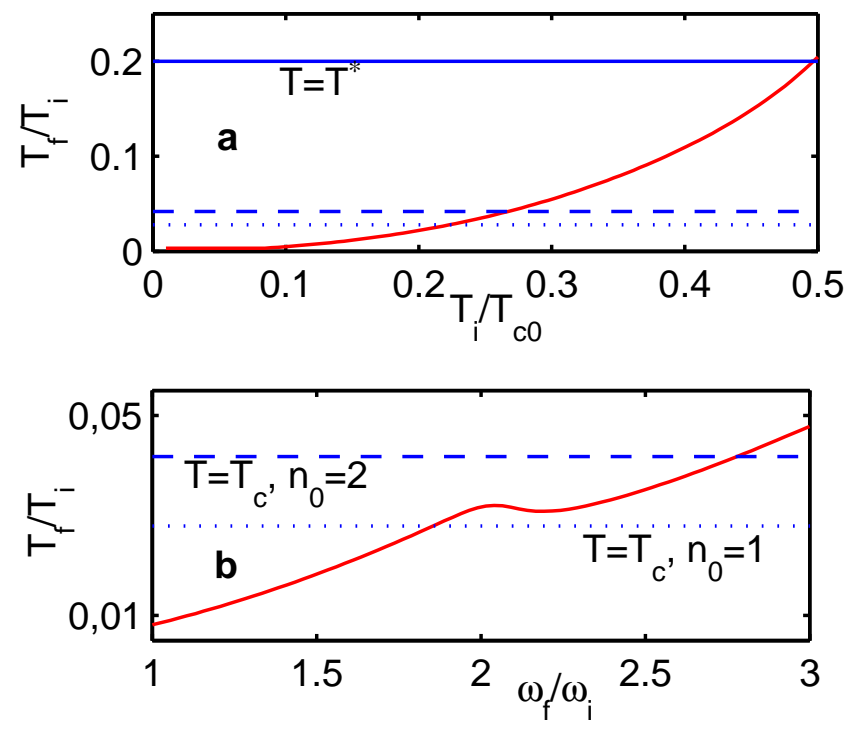